\title{
Bottomonia correlators and spectral functions at zero and finite temperature
}

\author{
K. Petrov*, \\ Physics Department, Brookhaven National Laboratory, Upton, NY, 11973, USA \\ E-mail: petrov@bnl.gov
}

\section{A. Jakovác}

Institute of Physics, BME Budapest, Budafoki Ãžút 8, H-1111 Budapest, Hungary

Antal.Jakovac@cern.ch

\section{P. Petreczky}

Physics Department, Brookhaven National Laboratory, Upton, NY, 11973, USA petreczkabnl.gov

\section{A. Velytsky}

Department of Physics and Astronomy, UCLA, Los Angeles, CA 90095-1547, USA

velephysics.ucla.edu

We present preliminary studies of bottomonia spectral functions at zero and finite temperature using quenched anisotropic lattices. The heavy quark is treated within Fermilab approach. We find no modification of the $\eta_{b}$ and $\Upsilon$ states up to temperatures $2.3 T_{c}$ while our study suggest dissolution of $\chi_{b}$ state at $1.15 T_{c}$.

PACS: $11.15 . \mathrm{Ha}, 11.10 . \mathrm{Wx}, 12.38 . \mathrm{Mh}, 25.75 . \mathrm{Nq}$

$23^{\text {rd }}$ International Symposium on Lattice Field Theory

Trinity college, Dublin

July 23-29, 2005

\footnotetext{
* Speaker.
} 


\section{Introduction}

Long ago it was conjectured by Matsui and Satz [1] that melting of different quarkonia states due to color screening can signal Quark Gluon Plasma formation in heavy ion collisions. The problem of quarkonia dissolution has been studied using potential models [3, 2, 4, 5, 6, 7]. However it is very unclear if such models are valid at the finite temperature [8]. Therefore the problem of dissolution of different quarkonia states should be studied in terms of the corresponding meson spectral functions. For charmonium such studies appeared only recently and suggested, contrary to potential models, that $J / \psi$ and $\eta_{c}$ survive till temperatures as high as $1.6 T_{c}[9,10,11]$. It has been also found that $\chi_{c}$ melts at temperature of about $1.1 T_{c}$ [11]. It is expected that there is a hierarchy of dissolution temperatures for different quarkonia, states corresponding to hierarchy of their sizes, i.e. we expect a sequential dissolution pattern where larger states dissolve at smaller temperatures. In this contribution we present our preliminary study of bottomonia spectral functions and correlators at finite temperature.

\section{Lattice correlators and spectral functions}

In our lattice investigation we calculate correlators of point meson operators of the form $\bar{q} \Gamma q$, where $\Gamma$ is one of the Dirac matrices which fixes the quantum number of the channel. We consider pseudo-scalar, vector, scalar, and axial-vector channels which correspond to $\eta_{b}, \Upsilon, \chi_{b 0}$ and $\chi_{b 1}$ respectively. The Euclidean time correlator $G(\tau, T)$ we calculate on the lattice is an analytic continuation of the real time correlator, $G(\tau, T)=D^{>}(-i \tau, T)$. Due to this relation the meson spectral function, defined as

$$
\sigma(\omega, T)=\frac{D^{>}(\omega, T)-D^{<}(\omega, T)}{2 \pi}
$$

is related to the Euclidean correlator through the integral representation

$$
\begin{aligned}
& G(\tau, T)=\int d \omega \sigma(\omega, T) K(\tau, \omega, T) \\
& K(\tau, \omega, T)=\frac{\cosh (\omega(\tau-1 /(2 T))}{\sinh (\omega /(2 T))} .
\end{aligned}
$$

Thus to reconstruct the spectral function from the lattice correlator $G(\tau, T)$ this integral representation should be inverted. Since the number of data points is less than number of degrees of freedom (which is $\mathscr{O}(100)$ for reasonable discretization of the integral ) spectral functions can be reconstructed only using the Maximum Entropy Method (MEM) [12]. In order to have sufficient number of data points either very fine isotropic lattices [11] or anisotropic lattices $[9,10]$ should be used. Another difficulty arises due to the large quark mass, discretization errors $\mathscr{O}\left(a m_{c, b}\right)$ are present in the heavy quark system. To remove these discretization errors we use the Fermilab approach $[13,14,15]$.

We performed calculation of bottomonia correlators in quenched QCD using anisotropic lattices and Wilson gauge action. We used three value of the gauge coupling $\beta=5.9,6.1,6.3$ and anisotropy $\xi=a_{s} / a_{t}=4$. When the lattice spacing is set using the Sommer scale $r_{0}=0.5 \mathrm{fm}$ the above gauge coupling correspond to lattice spacing $a_{t}^{-1}=5.88,8.18,10.89 \mathrm{GeV}$. Further details of the lattice action together with the parameters can be found in Ref. [15]. 

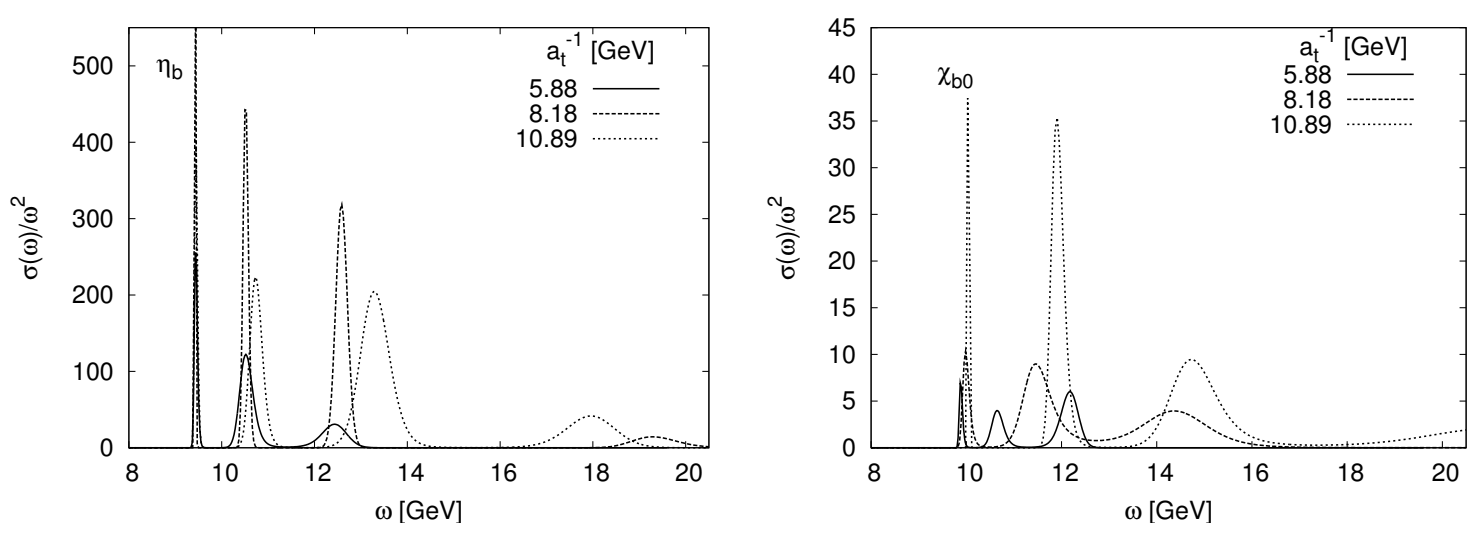

Figure 1: Bottomonia spectral functions in pseudo-scalar (left) and scalar (right) channel for different lattice spacing.

To be able to reconstruct the spectral functions high statistical accuracy for bottomonia correlators is required. This makes the calculations computationally intensive, so we performed them on a prototypes of RBRC QCDOC machine. It is a dedicated Lattice QCD machine developed by physicists from Columbia University, BNL, RIKEN and UKQCD. Three such machines, each reaching about 10TFlops peak performance, are currently installed at BNL and EPCC. Our prototypes were single-motherboard machine at about 50 GFlops peak. Such resources are still not adequate for the full QCD simulations, so we use quenched approximation, which is equivalent to neglecting quark loops. Typical statistics gathered was 500 to 1000 measurements, separated by 400 updates.

\section{Spectral functions at zero temperature}

Bottomonia spectral functions at $T=0$ are shown in Fig. 1 for the pseudo-scalar and scalar channels. The first peak is independent of the lattice spacing and corresponds to $\eta_{b}$ and $\chi_{b}$ respectively. The position of the higher peaks depends on the lattice spacing, so they cannot be interpreted as physical states. Similar results have been found for the vector and axial-vector channels.

\section{Correlators and spectral functions above deconfinement}

We would like to know what happens to different bottomonia states at temperatures above the deconfinement temperature $T_{c}$. With increasing temperature it becomes more and more difficult to reconstruct the spectral functions as both the number of available data points as well as the physical extent of the time direction (which is $1 / T$ ) decreases. Therefore it is useful to study the temperature dependence of bottomonia correlators first. From Eq. (2.3) it is clear that the temperature dependence of bottomonia correlators come from two sources: the temperature dependence of the spectral function and temperature dependence of the integration kernel $K(\tau, \omega, T)$. To separate out the trivial temperature dependence due to the integration kernel, following Ref [11] at each temperature we calculate the so-called reconstructed correlator

$$
G_{\text {recon }}(\tau, T)=\int_{0}^{\infty} d \omega \sigma(\omega, T=0) K(\tau, \omega, T)
$$



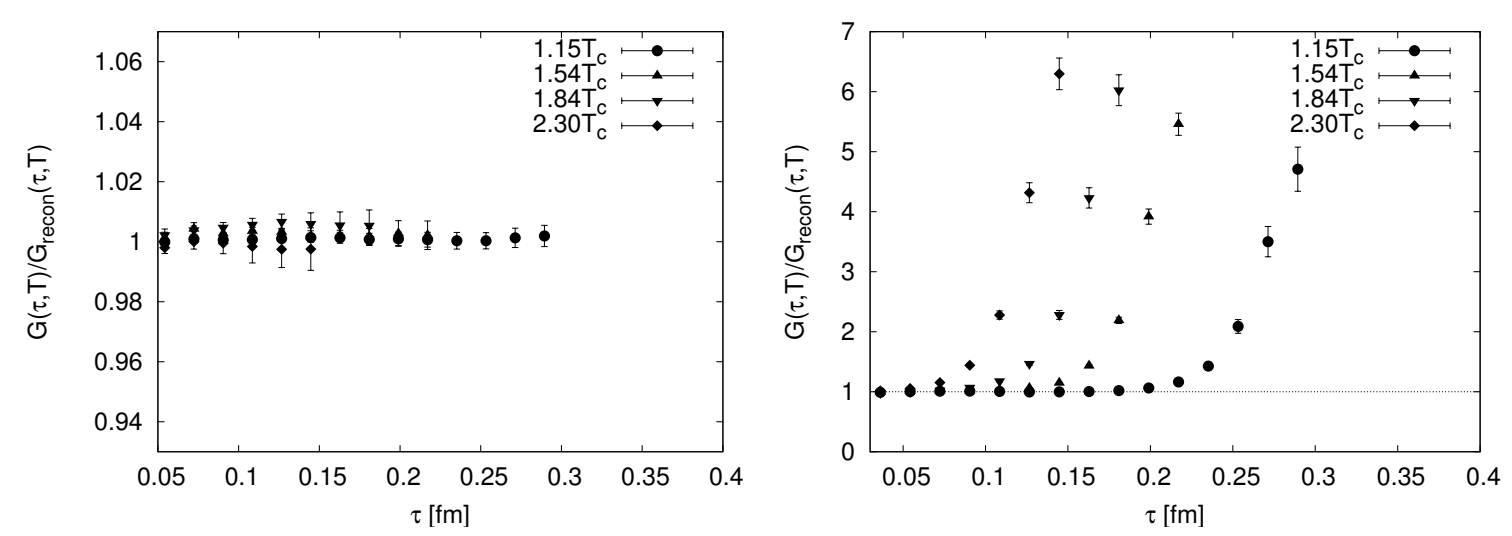

Figure 2: The ratio $G(\tau, T) / G_{\text {recon }}(\tau, T)$ for different temperatures at $\beta=6.3$.

Now if we assume that there is no temperature dependence in the spectral function - then the ratio of the original and the reconstructed correlator should be close to one, $G(\tau, T) / G_{\text {recon }}(\tau, T) \sim 1$. This way we can identify the cases when spectral function itself changes dramatically with temperature. This gives reliable information about the fate of quarkonia states above deconfinement. In Fig. 2 we show this ratio at $\beta=6.3$ for several temperatures corresponding to $N_{t}=32,24,20,16$ for pseudoscalar and scalar channels. As one can see from the figures for pseudo-scalar channel this ratio is close to one and shows no temperature dependence up to temperatures $T=2.3 T_{c}$. Thus we expect that $\eta_{b}$ will exist in the plasma phase with almost no modification of its properties. On contrary in the scalar channel the correlator shows large temperature dependence and $G(\tau, T) / G_{\text {recon }}(\tau, T)$ is far from unity already at $1.15 T_{c}$. Similar behavior of the correlators was found in the axial-vector channel. This suggest modification or eventually dissolution of $\chi_{b}$ above deconfinement. We find very similar temperature dependence of the correlators at larger lattice spacings corresponding to $\beta=5.9$ and 6.1. This suggest that despite possible lattice artifacts in the correlators and spectral functions medium modifications of bottomonia properties at finite temperature can be reliably understood.

More detailed information on different bottomonia states at finite temperature can be obtained by calculating spectral functions using MEM. The result of these calculation is show in Fig. 3. As expected the $\eta_{b}$ state survives in the deconfined phase with no mass shift. In the scalar channel the first peak becomes broader and smaller with increasing the temperatures, signaling dissolution of the $\chi_{b}$ state. This behavior of $\chi_{b}$ state is quite unexpected as it has similar size and binding energy as the $\eta_{c}$ and $J / \psi$ states. Thus in this respect, the expected sequential dissolution pattern of quarkonia states does not seem to hold.

\section{Acknowledgments}

This work was supported by U.S. Department of Energy under Contract No. DE-AC0298CH10886 and by SciDAC project. A.V. was partially supported by NSF-PHY-0309362. Authors used Columbia Physics System (CPS) with high-performance clover inverter written by P. Boyle and other parts written by RBC collaboration. Special thanks to C. Jung for his generous help with CPS. 

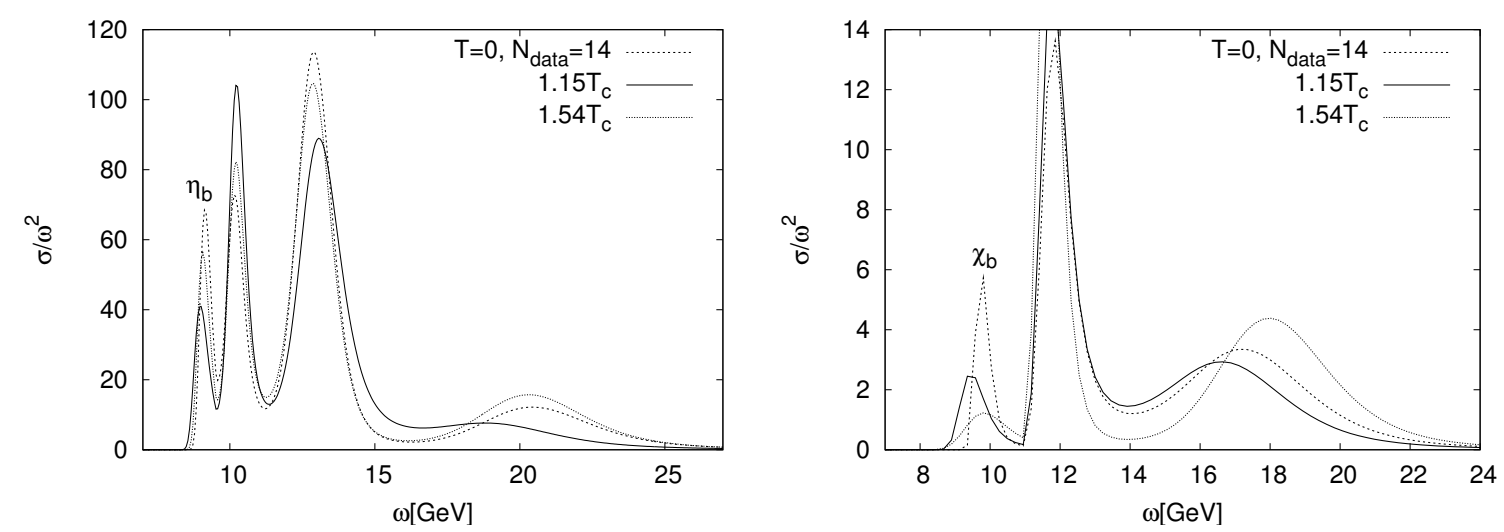

Figure 3: Bottomonia spectral functions in the pseudo-scalar (left) and scalar channel (right) above deconfinement for $\beta=6.3$.

\section{References}

[1] T. Matsui and H. Satz, Phys. Lett. B 178, 416 (1986).

[2] S. Digal, P. Petreczky and H. Satz, Phys. Lett. B 514, 57 (2001)

[3] F. Karsch, M. T. Mehr and H. Satz, Z. Phys. C 37, 617 (1988).

[4] S. Digal, P. Petreczky and H. Satz, Phys. Rev. D 64, 094015 (2001)

[5] E. V. Shuryak and I. Zahed, ' Phys. Rev. D 70, 054507 (2004)

[6] C. Y. L. Wong, hep-ph/0408020.

[7] Á. Mócsy, P. Petreczky, hep-ph/0411262

[8] P. Petreczky, contribution to Hard Probes 2004,

[9] T. Umeda, K. Nomura and H. Matsufuru, arXiv:hep-lat/0211003.

[10] M. Asakawa and T. Hatsuda, Phys. Rev. Lett. 92, 012001 (2004) [arXiv:hep-lat/0308034].

[11] S. Datta, F. Karsch, P. Petreczky and I. Wetzorke, Phys. Rev. D 69, 094507 (2004)

[12] M. Asakawa, T. Hatsuda and Y. Nakahara, Prog. Part. Nucl. Phys. 46, 459 (2001) [arXiv:hep-lat/0011040].

[13] A. X. El-Khadra, A. S. Kronfeld and P. B. Mackenzie, Phys. Rev. D 55, 3933 (1997) [arXiv:hep-lat/9604004].

[14] P. Chen, Phys. Rev. D 64, 034509 (2001)

[15] X. Liao and T. Manke, Phys. Rev. D 65, 074508 (2002) [arXiv:hep-lat/0111049]. 